\title{
Maternal ancestry analyses of red tilapia strains based on D- loop sequences of seven tilapia populations
}

\author{
Bingjie Jiang ${ }^{1}$, Jianjun Fu ${ }^{2}$, Zaijie Dong ${ }^{\text {Corresp., } 1,2}{ }^{2}$, Min Fang ${ }^{1}$, Wenbin Zhu ${ }^{2}$, Lanmei Wang ${ }^{2}$ \\ ${ }^{1}$ Wuxi Fisheries College, Nanjing Agricultural University, Wuxi, Jiangsu, China \\ Key Laboratory of Freshwater Fisheries and Germplasm Resources
Center of Chinese Academy of Fishery Sciences, Wuxi, Jiangsu, China \\ Corresponding Author: Zaijie Dong \\ Email address: dongzj@ffrc.cn
}

Background. Many tilapia species or varieties have been widely introduced and have become an economically important food fish in China. Information on the genetic backgrounds of these populations is deficient and requires more research, especially for red tilapia strains.

Methods. In the present study, displacement loop (D-loop) sequences were used to evaluate the genetic relationship and diversity of seven tilapia populations that are widely cultured in China; this was done specifically to speculate on the maternal ancestry of red tilapia strains. Three red tilapia varieties of Oreochromis ssp. , Chinese Taiwan (TW), Israel (IL), and Malaysia (MY) strains and other populations , including $O$. aureus (AR), $O$. niloticus (NL), $O$. mossambicus (MS), and GIFT strain of $O$. niloticus, were collected and analyzed in this study.

Results. A total of 146 polymorphic sites and 32 haplotypes of D-loop sequences were detected among 332 fish and four major haplotypes were shared among the populations. The TW and NL populations had a greater number of haplotypes (20 and 8 , respectively). The haplotype diversity ( $\mathrm{Hd}$ ) and nucleotide diversity $(\pi)$ of each population ranged from 0.234 to 0.826 , and 0 to 0.060 , respectively. The significant positive Tajima's $D$ value of neutral test were detected in the NL, IL, and MY populations $(P<0.05)$, which indicated these populations might have not experienced historical expansion. According to the pairwise $F$-statistics, highly significant genetic differentiations were detected among populations $(P<$ 0.01 ), with the exception of the IL and MY populations $(P>0.05)$. The nearest K2P genetic distance $(D=$ 0.014 ) was detected between the MS and TW populations, whereas, the farthest $(D=0.101)$ was found between the GIFT and AR populations. The results from the molecular variance analysis (AMOVA) showed that there was an extremely significant genetic variation observed among the populations $(P<0.01)$, which contained $63.57 \%$ of the total variation. In view of the genetic relationship of red tilapia strains with other populations, TW and IL were detected with more similar genetic structures related to MS, and MY was more genetically similar to GIFT (or NL), which could provide more genetic evidence for the red tilapia strains maternal ancestry. 


\section{Maternal ancestry analyses of red tilapia strains based \\ 2 on D-loop sequences of seven tilapia populations}

3

4

5 Bingjie Jiang ${ }^{1}$, Jianjun $\mathrm{Fu}^{2}$, Zaijie Dong ${ }^{1,2^{*}}$, Min Fang ${ }^{1}$, Wenbin $\mathrm{Zhu}^{2}$ and Lanmei Wang ${ }^{2}$

6

$7{ }^{1}$ Wuxi Fisheries College, Nanjing Agricultural University, Wuxi, Jiangsu, China

8

12 Corresponding Author:

13 Zaijie Dong

14 East Shanshui Road 9, Wuxi, Jiangsu, 214081, China

15

16

17
${ }^{2}$ Key Laboratory of Freshwater Fisheries and Germplasm Resources Utilization, Ministry of Agriculture and Rural Affairs, Freshwater Fisheries Research Center of Chinese Academy of Fishery Sciences, Wuxi, Jiangsu, China 
19

20

21

22

23

24

25

26

27

28

29

30

31

32

33

34

35

36

37

38

39

40

41

42

43

44

45

46

47

\section{ABSTRACT}

Background. Many tilapia species or varieties have been widely introduced and have become an economically important food fish in China. Information on the genetic backgrounds of these populations is deficient and requires more research, especially for red tilapia strains.

Methods. In the present study, displacement loop (D-loop) sequences were used to evaluate the genetic relationship and diversity of seven tilapia populations that are widely cultured in China; this was done specifically to speculate on the maternal ancestry of red tilapia strains. Three red tilapia varieties of Oreochromis ssp., Chinese Taiwan (TW), Israel (IL), and Malaysia (MY) strains and other populations, including O. aureus (AR), O. niloticus (NL), O. mossambicus (MS), and GIFT strain of $O$. niloticus, were collected and analyzed in this study.

Results. A total of 146 polymorphic sites and 32 haplotypes of D-loop sequences were detected among 332 fish and four major haplotypes were shared among the populations. The TW and NL populations had a greater number of haplotypes (20 and 8, respectively). The haplotype diversity (Hd) and nucleotide diversity $(\pi)$ of each population ranged from 0.234 to 0.826 , and 0 to 0.060 , respectively. The significant positive Tajima's D values of neutral test were detected in the NL, IL, and MY populations $(P<0.05)$, which indicated these populations might have not experienced historical expansion. According to the pairwise $F$-statistics, highly significant genetic differentiations were detected among populations $(P<0.01)$, with the exception of the IL and MY populations $(P>0.05)$. The nearest K2P genetic distance $(\mathrm{D}=0.014)$ was detected between the MS and TW populations, whereas, the farthest $(\mathrm{D}=0.101)$ was found between the GIFT and AR populations. The results from the molecular variance analysis (AMOVA) showed that there was an extremely significant genetic variation observed among the populations $(P<$ 0.01), which contained $63.57 \%$ of the total variation. In view of the genetic relationship of red tilapia strains with other populations, TW and IL were detected with more similar genetic structures related to MS, and MY was more genetically similar to GIFT (or NL), which could provide more genetic evidence for the red tilapia strains maternal ancestry.

\section{INTRODUCTION}

Tilapia as a common name has been applied to various cichlids from three distinct genera, which include Oreochromis, Sarotherodon, and Tilapia (Trewavas, 1983). The farmed tilapia production worldwide was over 6.4 million tons annually in 2015 and China is the largest tilapia 
50 producer in the world (FAO, 2016). Red tilapia is a name used for several different man-made 51 tilapia variants that have an attractive red coloration. These variants are the result of continuous selective breeding (Wohlfarth et al., 1990). Many farmers prefer to cultivate red tilapia since it is sought after in certain markets. Because of their high protein content, large size, high feed

54 55 conversion rate (FCR), rapid growth and palatability, red tilapia is the focus of major farming efforts in China (Romana-eguia et al., 1999).

In recent years, due to the increasing demand in the market, many red tilapia populations have been imported and cultured in Chinese farms. However, genetic introgression of those varieties is commonly detected because of their inter-population hybridization breeding. Growth and color separation often occurs in this practice, which greatly affects the promotion and marketing of red tilapia. In China, the genetic diversity studies of the tilapia populations based on molecular markers were carried out in the tilapia populations using TRAP (Ma et al., 2012), microsatellites (Zhang et al., 2010) and ISSR (Zhong et al., 2012). Research on tilapia in other countries has mainly focused on growth and development (Lith et al., 2005), culture (Muendo et al., 2006), and breeding (Fujimura et al., 2010). The information on genetic diversity and the genetic ancestries of red tilapia is lacking. The origin of the red tilapia was generally thought to be attributed to the cross-breeding of the mutant reddish-orange $O$. mossambicus and other populations including O. aureus, O. niloticus, and O. hornorum (Wohlfarth et al., 1990; Sandeep et al., 2012), but the specific source of the certain strains is ambiguous and the ancestries of the three strains widely cultivated in China are uncharted (Zhu et al., 2017).

The D-loop sequences are non-coding regions of mitochondrial DNA (mtDNA), with a high rate of evolution and no recombination, which becomes one of the most commonly used mtDNA sequences for addressing the evolutionary relationship of close relatives and/or subspecies (Murgra et al., 2002). At present, the D-loop sequences have been widely used in genetic analyses for aquaculture species, especially related to the genetic structure (Ryota et al., 2002), genetic differentiation (Brown et al., 2002), species validities (Tang et al., 2007), phylogeny and molecular differentiation (Ekerette et al., 2018). In this study, D-loop sequences were used to evaluate the genetic diversity of seven tilapia populations and to further estimate the maternal ancestry of three strains of red tilapia.

\section{MATERIALS AND METHODS}




\section{Sample collection}

82 The sampling scheme and experimental protocols were approved by the Bioethical Committee of 83 Freshwater Fisheries Research Center (FFRC) of the Chinese Academy of Fishery Sciences (CAFS) 84 (BC2013863, 9/2013). Experimental fish were sampled from seven populations, including the 85 three red tilapia strains of Oreochromis spp., Chinese Taiwan (TW), Israel (IL), and Malaysia 86 (MY) strains, and other populations of tilapia, including the GIFT strain of $O$. niloticus, $O$. 87 aureus (AR), O. niloticus (NL), O. mossambicus (MS). The TW and IL populations were 88 transferred from Fujian Province, China in 2014, and the MY population was introduced from 89 Malaysia in 2009 (Yang et al., 2015). All tilapia populations were domesticated and bred in an 90 experimental aquaculture farm in Wuxi (Jiangsu Province, China). The methods for handling the animals and the experimental procedures conducted were in accordance with the guidelines for the care and use of animals for scientific purposes set by the Ministry of Science and Technology, Beijing China (No.398, 2006). Forty-eight fin clips were sampled from each population and soaked in absolute ethanol until the DNA was extracted.

\section{DNA extraction and amplification}

Genomic DNA was extracted using the phenol-chloroform method (Sambrook, 2001). The integrity was detected by $1 \%$ agarose gel electrophoresis. The purity and concentration of the DNA was detected using the NanoDrop spectrophotometer. The DNA concentration of each sample was adjusted to about $20 \mathrm{ng} / \mu \mathrm{L}$ and kept under $-20^{\circ} \mathrm{C}$ until ready to use.

Primers of the D-loop were designed according to the complete sequences of tilapia mtDNA (Accession NO: NC_014060) from the National Center for Biotechnical Information (NCBI). The D-loop sequences of 867 bp was amplified using a primer pair (sense primer: 5'CTACTTCTTCCTCTTCCTTGT-3', anti-sense primer: 5'-TCCGTCTTAACATCTTCAGT-3'), which was synthesized by Sangon Biotech (Shanghai) Co.Ltd. The PCR amplification was performed on an Eppendorf Mastercycler Pro 384 PCR thermocycler (Eppendorf, Germany). Amplifications were performed in a volume of $50 \mu \mathrm{L}$, containing $5 \mu \mathrm{L} 10 \times$ PCR Buffer, $3 \mu \mathrm{L}$ $\mathrm{MgCl}_{2}(0.25 \mathrm{mM}), 4 \mu \mathrm{L}$ dNTPs $(2.5 \mathrm{mM}), 1 \mu \mathrm{L}$ Taq polymerase $(2.5 \mathrm{U} / \mu \mathrm{L}), 1 \mu \mathrm{L}$ of each primer $(10 \mu \mathrm{M}), 2 \mu \mathrm{L}$ genomic DNA $(20 \mathrm{ng} / \mu \mathrm{L})$, and $33 \mu \mathrm{L}$ DNase/RNase-free deionized water. PCR amplification was performed under the following conditions: pre-denaturing for $2 \mathrm{~min}$ at $94{ }^{\circ} \mathrm{C}$; 35 cycles of denaturing for $40 \mathrm{~s}$ at $94{ }^{\circ} \mathrm{C}$, annealing for $55 \mathrm{~s}$ at $55^{\circ} \mathrm{C}$, prolonging $1 \mathrm{~min}$ at $72{ }^{\circ} \mathrm{C}$; final prolonging for $10 \mathrm{~min}$ at $72{ }^{\circ} \mathrm{C}$; and then held at $12{ }^{\circ} \mathrm{C}$. Subsequently, the reaction products 
112 were detected using 1\% agarose gel electrophoresis, and the bidirectional sequencing was carried

113 out with the ABI3730XL sequencing instrument of Shanghai Majorbio Company.

114 Sequence arrangement and data analysis

115 The sequences were edited using the BioEdit version 7.0.9 software (Hall, 1998). To ensure

116 accuracy, all DNA fragments were sequenced in two directions, and the assembled sequences

117 were manually checked to prevent the ambiguity of the base or sequencing error. After the

118 completion of the splicing, all sequences were used for homologous alignment and length

119 determination by the BioEdit version 7.0.9 software.

120 After comparing and sorting the D-loop sequences, the fuzzy sequences were deleted and

121332 homologous sequences were obtained upon completion. Genetic variation parameters were

122 calculated by the DnaSP 5.1 software (Librado et al., 2009), including polymorphic (segregating)

123 sites $(\mathrm{S})$, number of haplotypes $(\mathrm{h})$, haplotype diversity $(\mathrm{Hd})$, nucleotide diversity $(\pi)$, average

124 number of nucleotide differences (k) and Tajima's D. For phylogenetic analysis, MEGA 5.05

125 software (Kimura, 1980; Tamura et al., 2011) was utilized to calculate the Kimura 2-parameter

126 (K2P) distance among populations, and to construct unweighted pair group methods with an

127 arithmetic (UPGMA) dendrogram set with 1000 replications of bootstrapping. Arlequin 3.5

128 software (Excoffier, 2010) was used to analyze the nucleotide composition, $F$-statistics $\left(F_{\mathrm{ST}}\right)$ and

129 the analysis of molecular variance (AMOVA) among seven tilapia populations. The Network 4.6

130 software (Polzin, 2003) was used to construct the network for haplotypes of D-loop sequences.

131

132 RESULTS

133 Variation and haplotype distribution of D-loop sequences in tilapia

134 The nucleotide frequencies of the seven tilapia populations were consistent, and clearly, the rate 135 of $\mathrm{A}+\mathrm{T}$ (the average value is $64.3 \%$ ) was higher than in $\mathrm{C}+\mathrm{G}$ (the average value is $35.7 \%$ ). By

136 sequence alignment, a total of 32 haplotypes were found in the D-loop sequences and deposited

137 in the GenBank database under the accession numbers MH515150-MH515185 (except for

138 MH515152, MH515172, MH515175, and MH515182). Different numbers of haplotypes (from 1

139 to 20) were detected among the populations (Table 1). Four of these haplotypes were shared

140 haplotypes (Hap_2, Hap_22, Hap_23, and Hap_24), of which 2 haplotypes were composed of

141 NL and GIFT populations (Hap_22, Hap_23), while the others were unique to each population. 
142 The 4 dominant haplotypes in all individuals were Hap_2, Hap_22, Hap_24, and Hap_26

143 accounting for $34.90 \%, 12.30 \%, 14.50 \%$, and $14.50 \%$, respectively.

144 Genetic diversity and genetic distance among seven tilapia populations

145 The genetic diversity parameters of the tilapia populations based on D-loop sequences are shown 146 in Table 2. A total of 146 polymorphism sites were found, the overall haplotype diversity (Hd) of 147 the tilapia populations was 0.817 , and each population ranged from 0 to 0.834 . The average 148 number of nucleotide differences $(K=0-47.32)$ and nucleotide diversity $(\pi=0-0.060)$ were 149 determined. Among them, the AR population had the lowest genetic diversity $(\mathrm{Hd}=0, \pi=0)$, 150 the TW and NL populations had a higher haplotype diversity ( $\mathrm{Hd}=0.834,0.826$, respectively), 151 and the highest nucleotide diversity $(\pi)$ was detected in the NL population $(\pi=0.060)$. Tajima's

152 test indicated that the Tajima's D value of the TW, GIFT, and MS populations were negative, and 153 other populations were positive. Among them, NL and MS reached a significant level $(P<0.05)$, 154 GIFT, IL and MY populations reached extremely significant levels $(P<0.01)$.

155 The pairwise genetic distance was calculated using the Kimura 2-parameter (K2P) model 156 among seven tilapia populations (Table 3, below diagonal). The inter-population distances 157 among seven populations ranged from 0.014 to 0.101 . The closest inter-population distance 158 (0.014) was between the MS and TW populations, while the furthest inter-population distance 159 (0.101) was between the GIFT and AR populations. In this study, the UPGMA tree based on the 160 K2P genetic distance was shown in Figure 1. According to the phylogenetic tree, the haplotype 161 was obviously divided into two branches (the AR population was separated from other six 162 populations). In addition, the MS and TW populations were clustered and then clustered with the 163 IL population; GIFT and MY populations were clustered and then clustered with the NL 164 population.

\section{Genetic differentiation among tilapia populations}

166 The results of the analysis of molecular variance (AMOVA) were shown in Table 4. Based on 167 the results of the genetic differentiation analysis, the variance percentage of genetic variation 168 among populations in total variance was 63.57\%, and a high degree of inter-population 169 differentiation was observed among populations $(F \mathrm{st}=0.636, P<0.01)$. The seven populations 170 were divided into 2 groups consisting of wild-type or breeding populations, and red tilapia. The 171 genetic differentiation index among populations within groups accounted for $0.633(P<0.01)$.

172 The pairwise $F$-statistics values $(F$ st) of the seven tilapia populations (Table 3, upper right 
173 corner) showed that the pairwise genetic differentiations among the populations were very

174 significant $(P<0.01)$, except among the MY and IL populations $(P>0.05)$.

175 Haplotype network of D-loop sequences in tilapia populations

176 The median-joining (MJ) network diagram of D-loop haplotypes was described in Figure 2. The

177 MJ network presented a star-like profile, which was linked to many haplotypes from different

178 regions, and the shared haplotypes and dominant haplotypes were clearly defined. Obviously,

179 three shared haplotypes were composed of two populations (GIFT and NL, MY and IL), one

180 shared haplotype was composed of six populations (except AR) and a dominant haplotype was

181 composed of an AR population.

182 Maternal ancestry of red tilapia strains

183 According to the genetic distances among the tilapia populations (Table 3, below diagonal), it

184 was calculated that TW, IL red tilapia, and MS populations had the closest genetic distance,

185 which were 0.014 and 0.032 , respectively. The genetic distance between MY red tilapia and the

186 GIFT population was the closest (0.034). Moreover, the UPGMA tree clearly divided the tested

187 samples of red tilapia into two independent branches. TW and IL were clustered into one branch,

188 and then clustered with MS, while MY divided into another branch and clustered with GIFT and

189 NL.

190

191 DISCUSSION

192 Genetic diversity and population dynamics

193 In the present study, the content of A+T (64.3\%) in tilapia D-loop sequences was higher than the 194 content of $\mathrm{G}+\mathrm{C}(35.7 \%)$, which was in line with the distribution characteristics of the base 195 content in the D-loop (control region) of many fishes (Broughton et al., 2001). In D-loop 196 sequences of seven tilapia populations, 146 polymorphic (segregating) sites (S) and 32

197 haplotypes were detected, suggested that D-loop sequences could be an effective marker for 198 genetic diversity analysis for tilapia populations. Overall, the tilapia populations showed high

199 haplotype diversity and nucleotide diversity in this study, indicating the populations that contain 200 an abundance of genetic resources for further use in breeding or practice. Specifically, NL had 201 the higher genetic diversity $(\mathrm{Hd}>0.5, \pi>0.005)$, which was consistent with other research 202 using microsatellite markers (Romana-eguia et al., 2004; Yang et al., 2011), and isozymes (Zhao 203 et al., 1997). It was speculated that the original NL population introduced in China was larger 
204 and it had a potential for further selective breeding. The genetic diversity of the red tilapia 205 populations (TW, IL, and MY) was higher than the other tilapia populations, probably due to its 206 genetic background of cross-breeding. However, the genetic diversity of the AR population was

207

208

209

210

211

212

213

214

215

216

217

218

219

220

221

222

223

224

225

226

227

228

229

230

231

232

233

234 the lowest ( $\mathrm{Hd}=0, \pi=0)$, which was found to be similar to the results of the previous reports assessed by RAPD (Xia et al., 1999), microsatellite probes (Wang et al., 2000), and mtDNA restriction enzyme analysis (Cao et al., 1997). This may be due to the small population size introduced in China as well as mass breeding over generations, which might result in a decline in the genetic polymorphism of the population. The genetic purity of the AR population was adverse to further selective breeding and it is necessary to introduce the AR population again in order to improve its genetic diversity and reduce its depression as a result of inbreeding. In contrast, the purity of this population also could be used for inhybridization with other populations or strains. The GIFT and MS populations were also detected with low diversity parameters $(\mathrm{Hd}<0.5, \pi<0.005)$, indicating that its population may have recently experienced a bottleneck or founder effect produced by minority populations (Grant et al., 1998). The high purity of the AR, GIFT, and MS populations has great significance that the excellent economic traits obtained through long-term multi-generation breeding could insure the stability inherited through the genetic selection process. Therefore, AR, GIFT, and MS were often used as parents to breed red tilapia and stabilize the excellent traits in the red tilapia populations. The Tajima's D value for some red tilapia (TW) and wild-type or breeding populations (MS, GIFT) were negative, which is probably due to the population expansion caused by larger scale breeding after smaller breeder population produced a family selection in the hatchery.

\section{Genetic relationships of tilapia populations}

The red tilapia stocks were reported with different genetic sources that originated from $O$. mossambicus, O. aureus, or O. niloticus (Wohlfarth et al., 1990; Sandeep et al., 2012). In this study, four relative tilapia populations were used for exploring the maternal ancestors for three strains of red tilapia. The MJ network of D-loop sequences for haplotypes in the tilapia populations, which were divided into three different major regions and red tilapia populations, presented in two regions. Four dominant haplotypes were shared by the seven populations, where Hap_24 was a shared by MY and IL populations, Hap_22 and Hap_23 were shared by GIFT and NL populations, and Hap_2 was shared by six populations except for the AR population. It was suggested that six populations (except AR) may originate from similar maternal ancestors. 
The analysis of molecular variance (AMOVA) by grouping and non-grouping showed that

236

237

238

239

240

241

242

243

244

245

246

247

248

249

250

251

252

253

254

255

256

257

258

259

260

261

262

263

264

265 the main genetic variation was derived from inter-population, which is similar to the results of Habib et al. (2010), the low variance within the population and high variance among populations was reported among Channa fishes. The fixation index $(F$ st) was commonly used to examine the genetic variation of populations and the contribution of this variation to genetic differentiation (Holsinger et al., 2009). Significant $F$ st values ( $F$ st $>0.25, P<0.01$ ) were found in this study, which demonstrated that a higher level of genetic differentiation exists among tilapia populations except for red tilapia. The results indicated that red tilapia populations may have evolved independently after separating from a common ancestor, but that those three strains of red tilapia were closed.

\section{Analysis of maternal ancestry of red tilapia}

While the maternal ancestors of the existing three strains of red tilapia are not well documented, their derivation is generally attributed to the crossbreeding of the mutant reddish-orange Oreochromis mossambicus with other species including O. aureus, $O$. niloticus, and $O$. hornorum (Wohlfarth et al., 1990; Sandeep et al., 2012). Mitochondrial DNA (mtDNA) has the characteristics of maternal inheritance, simple structure, and rapid evolution. Therefore, the phylogenetic tree constructed by mtDNA can directly reflect the origin of the maternal ancestry (Cann, 1994).

Based on the K2P genetic distances among seven tilapia populations, two branches were constructed in the UPGMA dendrogram and it was speculated that these populations might be derived from two different primary maternal ancestors, which was consistent with the results of the MJ network. Specifically, three strains of red tilapia might derive from different maternal origin, MS and GIFT (or NL) populations, respectively. The TW and IL populations were closely related to each other and were clustered with MS, which was confirmed that two strains of red tilapia were produced from local crossbreeding of the rare, mutant-colored (reddishorange) female O.mossambicus (Wohlfarth et al., 1990). The GIFT strain was selected from four O. niloticus strains imported directly from Africa and four strains widely cultivated in Asia (Eknath et al., 1993). The K2P genetic distance between MY red tilapia and the GIFT population was relatively small $(\mathrm{D}=0.034)$, speculating that MY population was probably bred with the GIFT population or that MY and GIFT populations might come from the similar, artificially selected NL population. In addition, the degree of genetic differentiation between red tilapia (IL, 
266 TW, and MY) and the breeding source populations (MS, GIFT or NL) were relatively small, 267 demonstrating that a close genetic relationship was maintained between the breeding varieties 268 and breeding source populations; this highly homology was related to the characteristics of 269 maternal inheritance and non-recombination of mtDNA (Mabuchi, 2010).

270

\section{CONCLUSIONS}

272 In this study, we used the D-loop sequences to estimate the genetic structures of seven tilapia 273 populations mainly cultured in China. Furthermore, we analyzed the maternal ancestry of three 274 strains of red tilapia, which provides more basic data for the reasonable protection and further 275 utilization of tilapia populations in the future. In brief, the IL and TW red tilapia strains may 276 derive from the $O$. mossambicus population, whereas the MY red tilapia was probably derived 277 from GIFT or $O$. niloticus.

278

279

280

281

282

283

284

285

286

287

288

289

290

291

292

293

294

\section{REFERENCES}

Broughton RE, Milam JE, Roe BA. 2001. The complete sequence of the zebrafish (Danio rerio) mitochondrial genome and evolutionary patterns in vertebrate mitochondrial DNA. Genome Research 11(11):158-1967

Brown KH, Thorgaard GH. 2002. Mitochondrial and nuclear inheritance in an androgenetic line of rainbow trout, Oncorhynchus mykiss. Aquaculture 204(3):323-335

Cann RL, Stoneking M, Wilson AC. 1994. Mitochondrial DNA and human evolution. Journal of Bioenergetics and Biomembranes 26(3):251

Cao Y, Xia DQ. 1997. Studies on the genetic variation in mitochondrial DNA of O. niloticus and O. aureus. Journal of Fisheries of China 21:360-365

Ekerette EE, Ikpeme EV, Udensi OU, Ozoje MO, Etukudo OM, Umoyen AJ, Durosaro SO, and Wheto M. 2018. Phylogenetics and molecular divergence of tilapia fish (Oreochromis species) using mitochondrial D-loop and cytochrome b regions. American Journal of Molecular Biology 8:39-57

Eknath AE, Tayamen MM, Palada M, Casayuran-Danting J, Reyes RA, Dioisio E. 1993. Genetic improvement of farmed tilapia: a complete diallele cross between four African and Asian strains of Nile tilapia (Oreochromis niloticus). Aquaculture 111(1-4): 297. 
296 Excoffier L, Lischer HE. 2010. Arlequin suite ver.3.5: a new series of programs to perform 297 population genetics analyses under Linux and Windows. Molecular Ecology Resources $298 \quad 10(3): 564-567$

299 Food and Agriculture Organization of the United Nations (FAO). 2016. Fisheries and 300 aquaculture department, Global Aquaculture Production.

301 Fujimura K, Okada N. 2010. Development of the embryo, larva and early juvenile of Nile tilapia 302 Oreochromis niloticus (Pisces:Cichlidae) development staging system. Development, $303 \quad$ Growth and Differentiation 49:301-324

304 Grant WS, Bowen BW. 1998. Shallow population histories in deep evolutionary lineages of 305 306 307 308 309 310 Heredity 89 (5):415-426

Habib M, Lakra WS, Vindhya M, Praveen K, Barman AS, Akanksha S, Kuldeep KL, Peyush P and Asif AK. 2010. Evaluation of cytochrome b mtDNA sequences in genetic diversity studies of Channa marulius (Channidae: Perciformes). Molecular Biology Reports 6:41-49

Hall TA. 1998. BioEdit: a user-friendly biological sequence alignment editor and analysis program for windows 95/98/NT. Nucleic Acids Symposium Series 41:95-98

Holsinger KE, Bruce SW. 2009. Genetics in geographically structured populations: defining, estimating and interpreting FST. Nature Reviews Genetics 10:639-650

Kimura M. 1980. A simple method for estimating evolutionary rates of base substitutions through comparative studies of nucleotide sequences. Molecular Biology and Evolution 16:111-120

Librado P, Rozas J. 2009. DnaSP v5: a software for comprehensive analysis of DNA polymorphism data. Bioinformatics 25(11):1451-1452

Lith D, Cherop L, Munguti J, Chhorn L. 2005. Growth and economic performance of Nile tilapia (Oreochromis niloticus L.) fed on two formulated diets and two locally available feeds in fertilized ponds. Aquaculture Research 36:746-752

Ma QN. 2012. Screening of TRAP markers and analysis of genetic diversity in red tilapia. Thesis for M.S. Nanjing Agricultural University.

Mabuchi K, Senou H, Nishida M. 2010. Mitochondrial DNA analysis reveals cryptic large-scale invasion of non-native genotypes of common Carp (Cyprinuscarpio) in Japan. Molecular Ecology 17(3):796-809 
327 Muendo PN, Milstein A, Dam A, Gamal NE, Stoorvogel JJ, Verdegem MCJ. 2006. Exploring

328

329

330

331

332

333

334

335

336

337

338

339

340

341

342

343

344

345

346

347

348

349

350

351

352

353

354

355

356 the trophic structure in organically fertilized and feed-driven tilapia culture environments using multivariate analyses. Aquaculture Research 37:151-163

Murgra R, Tola G, Archer SN, Vallerga S, Hirano J. 2002. Genetic identification of grey mullet species (Mugilidae) by analysis of mitochondrial DNA sequence: application to identify the origin of processed ovary products (Bottarga). Marine Biotechnology 4(2):119-26

Polzin T, Daneshm, SV. 2003. On Steiner trees and minimum spanning trees inhypergraphs. Operations Research Letters 31(1):12-20

Romana-eguia MRR, Eguia RV. 1999. Growth of five Asian red tilapia strains in saline environments. Aquaculture 173:161-170

Romana-eguia MRR, Ikeda M, Basiao ZU, Taniguchi N. 2004. Genetic diversity in farmed Asian Nile and red hybrid tilapia stocks evaluated from microsatellite and mitochondrial DNA analysis. Aquaculture 236(1-4):131-150

Ryota Y, Akira G. 2002. Phylogeography of a freshwater Sculpin, Cottusnozawae from the northeastern part of Honshu Island, Japan. Ichthological Research 49(2):147-155

Sambrook J, Russell DW. 2001. Molecular cloning: A Laboratory Manual. 3rd ed. Cold Spring Harbor, NY, USA: Cold Spring Harbor Laboratory

Sandeep M, Sun F, Liu F, Li J, David PB, Yue GH. 2012. Novel polymorphic microsatellites from Florida red tilapia and cross-species amplification in Mozambique and Nile tilapia. Genet 91:e97-e99

Tamura K, Peterson D, Peterson N, Stecher G, Nei M, Kumar S. 2011. MEGA5: molecular evolutionary genetics analysis using maximum likelihood, evolutionary distance, and maximum parsimony methods. Molecular Biology and Evolution 28:2731-2739

Tang WQ, Hu XL, Yang JQ. 2007. Species validities of Coilia brachygnathus and C. nasus taihuens is based on sequence variations of complete mtDNA control region. Biodiversity Science 15(3):224-231

Trewavas E. 1983Tilapiine fishes of the genera Sarotherodon, Oreochromis and Danakilia. Ithaca New York: Cornell University Press.583p

Wang JK, Xia DQ and Wu TT. 2000. Studies on genetic polymorphism of O. aureus of founder in China with DNA finger printing. Journal of Nanjing Agricultural University 23(3):61-63

Peer) reviewing PDF | (2019:01:34177:2:0:REVIEW 17 Apr 2019) 
357 Wohlfarth GW, Rothbard S, Hulata G, Szweigman D. 1990. Inheritance of red body coloration 358 in Taiwanese tilapias and in Oreochromis mossambicus. Aquaculture 84:219-234

359 Xia DQ, Cao Y, Ting WU, Wang T. 1999. A study on genetic variation of tilapias fish with 360 RAPD analysis and its application to heterosis. Journal of Fisheries of China 23(1):27-32

361

362

363

364

365

366

367

368

369

370

371

372

373

374

375

376

377
Yang H, Li DY, Cao X, Zou ZY, Xiao W, Zhu JL. 2011. Genetic potential analysis of six tilapia populations by microsatellite DNA markers. Hereditas (Beijing) 33:768-7

Yang H, Zhu WB, Dong ZJ, Li F, Gong CP, Liu N, Yuan XH. 2015. Morphological variation analysis of three populations of red tilapia. Journal of Shanghai Ocean University 24(5):678-684

Zhang YD, Gan X, Tang ZS, Chen Z, Su XH, Tang ZY, Li LP, Lin Y. 2010. Analysis of genetic diversity in six tilapia populations. Journal of Northwest A and F University 38(10):58-66

Zhao JL, Li SF, Li CH, Li JL. 1997. Study of biochemical genetic marker of different strains of Nile tilapia Oreochromis niloticus. Journal of Shanghai Fisheries University 6:166-70

Zhong JX, Li JQ, Zhong R, Huang HG, Yang SC. 2012. ISSR analysis on genetic relationships of five Nile tilapia O. niloticus populations. Journal of Fujian Fisheries 34(5):349-353

Zhu WB, Yang H, Yuan XH, Dong ZJ, Fu JJ, Wang LM. 2017. High genetic diversity and differentiation in three red tilapia stocks revealed by microsatellite DNA marker analysis. Aquaculture International 25(22):1-10 
Table $\mathbf{1}$ (on next page)

Distribution of the D-loop haplotypes in tilapia populations 
Table 1 Distribution of the D-loop haplotypes in tilapia populations

\begin{tabular}{|c|c|c|c|c|c|c|c|c|c|}
\hline \multirow{2}{*}{ Haplotype } & \multirow{2}{*}{ Accession } & \multicolumn{4}{|c|}{ Wild-type or breeding populations } & \multicolumn{3}{|c|}{ Red tilapias } & \multirow{2}{*}{ Sum } \\
\hline & & NL & AR & MS & GIFT & TW & MY & IL & \\
\hline Hap_1 & MH515150 & & & 1 & & & & & 1 \\
\hline Hap_2 & MH515151 & 5 & & 46 & 1 & 18 & 18 & 28 & 116 \\
\hline Hap_3 & MH515153 & & & & & 1 & & & 1 \\
\hline Hap_4 & MH515154 & & & & & 1 & & & 1 \\
\hline Hap_5 & MH515155 & & & & & 1 & & & 1 \\
\hline Hap_6 & MH515156 & & & & & 7 & & & 7 \\
\hline Hap_7 & MH515157 & & & & & 1 & & & 1 \\
\hline Hap_8 & MH515158 & & & & & 1 & & & 1 \\
\hline Hap_9 & MH515159 & & & & & 3 & & & 3 \\
\hline Hap_10 & MH515160 & & & & & 1 & & & 1 \\
\hline Hap_11 & MH515161 & & & & & 1 & & & 1 \\
\hline Hap_12 & MH515162 & & & & & 1 & & & 1 \\
\hline Hap_13 & MH515163 & & & & & 1 & & & 1 \\
\hline Hap_14 & MH515164 & & & & & 1 & & & 1 \\
\hline Hap_15 & MH515165 & & & & & 1 & & & 1 \\
\hline Hap_16 & MH515166 & & & & & 1 & & & 1 \\
\hline Hap_17 & MH515167 & & & & & 1 & & & 1 \\
\hline Hap_18 & MH515168 & & & & & 2 & & & 2 \\
\hline Hap_19 & MH515169 & & & & & 1 & & & 1 \\
\hline Hap_20 & MH515170 & & & & & 2 & & & 2 \\
\hline Hap_21 & MH515171 & & & & & 1 & & & 1 \\
\hline Hap_22 & MH515173 & 9 & & & 32 & & & & 41 \\
\hline Hap_23 & MH515174 & 3 & & & 14 & & & & 17 \\
\hline Hap_24 & MH515176 & & & & & & 29 & 19 & 48 \\
\hline Hap_25 & MH515177 & & & & & & & 1 & 1 \\
\hline Hap_26 & MH515178 & & 48 & & & & & & 48 \\
\hline Hap_27 & MH515179 & & & & & & 1 & & 1 \\
\hline Hap_28 & MH515180 & 15 & & & & & & & 15 \\
\hline Hap_29 & MH515181 & 3 & & & & & & & 3 \\
\hline Hap_30 & MH515183 & 8 & & & & & & & 8 \\
\hline Hap_31 & MH515184 & 3 & & & & & & & 3 \\
\hline Hap_32 & MH515185 & 1 & & & & & & & 1 \\
\hline
\end{tabular}

2 
Table 2 (on next page)

Genetic diversity parameters of mtDNA D-loop sequence of seven tilapia populations 
1 Table 2 Genetic diversity parameters of mtDNA D-loop sequence of seven tilapia populations

\begin{tabular}{ccccccccc}
\hline & NL & AR & MS & GIFT & TW & MY & IL & Sum \\
\hline S & 116 & 0 & 6 & 70 & 101 & 71 & 74 & 146 \\
$\mathrm{~h}$ & 8 & 1 & 2 & 3 & 20 & 3 & 3 & 32 \\
$\mathrm{Hd}$ & 0.826 & 0 & 0.043 & 0.457 & 0.834 & 0.504 & 0.513 & 0.817 \\
$\pi$ & 0.060 & 0 & 0.0003 & 0.004 & 0.024 & 0.039 & 0.040 & 0.054 \\
$\mathrm{k}$ & 47.32 & 0 & 0.255 & 3.380 & 20.50 & 33.55 & 34.38 & 45.36
\end{tabular}

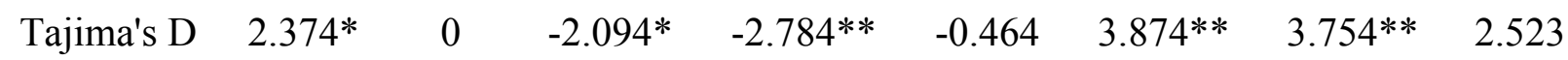

2 Notes: S, polymorphic sites; h, haplotypes; Hd, haplotype diversity; $\pi$, nucleotide diversity; $\mathrm{k}$,

3 average number of nucleotide differences; * means significant $(P<0.05)$; $* *$ means extremely

4 significant $(P<0.01)$. 


\section{Table 3(on next page)}

Pairwise K2P genetic distances (below diagonal) and fixation indexes (FST, above diagonal) among seven tilapia populations using D-loop 
1 Table 3 Pairwise K2P genetic distances (below diagonal) and fixation indexes $\left(F_{\mathrm{ST}}\right.$, above 2 diagonal) among seven tilapia populations using D-loop

\begin{tabular}{cccccccc}
\hline & NL & AR & MS & GIFT & TW & MY & IL \\
\hline NL & - & $0.612^{* *}$ & $0.639 * *$ & $0.333^{* *}$ & $0.463^{* *}$ & $0.181^{* *}$ & $0.263^{* *}$ \\
AR & 0.070 & - & $0.999^{* *}$ & $0.978^{* *}$ & $0.876^{* *}$ & $0.800^{* *}$ & $0.794^{* *}$ \\
MS & 0.076 & 0.093 & - & $0.971^{* *}$ & $0.128^{* *}$ & $0.612^{* *}$ & $0.379^{* *}$ \\
GIFT & 0.045 & 0.101 & 0.078 & - & $0.798^{* *}$ & $0.395^{* *}$ & $0.571^{* *}$ \\
TW & 0.073 & 0.095 & 0.014 & 0.070 & - & $0.369^{* *}$ & $0.133^{* *}$ \\
MY & 0.057 & 0.097 & 0.050 & 0.034 & 0.049 & - & 0.079 \\
IL & 0.064 & 0.095 & 0.032 & 0.050 & 0.036 & 0.042 & - \\
\hline
\end{tabular}

$3 \quad$ Note: $* *$ means extremely significant $(P<0.01)$. 


\section{Table 4 (on next page)}

Analysis of molecular variance (AMOVA) of 7 populations of tilapia mtDNA D-loop 
1 Table 4 Analysis of molecular variance (AMOVA) of seven tilapia populations based on D-loop sequences

\begin{tabular}{|c|c|c|c|c|c|}
\hline $\begin{array}{l}\text { Source of } \\
\text { variation }\end{array}$ & d.f. & Sum of squares & $\begin{array}{c}\text { Variance } \\
\text { components }\end{array}$ & $\begin{array}{l}\text { Percentage of } \\
\text { variation }\end{array}$ & $\begin{array}{c}\text { Fixation } \\
\text { Index }\end{array}$ \\
\hline \multicolumn{6}{|l|}{ No group } \\
\hline $\begin{array}{c}\text { Among } \\
\text { populations }\end{array}$ & 6 & 5848.14 & 20.31 & 63.57 & $0.636 * *$ \\
\hline $\begin{array}{c}\text { Within } \\
\text { populations }\end{array}$ & 325 & 3781.13 & 11.63 & 36.43 & \\
\hline Total & 331 & 9629.27 & 31.94 & & \\
\hline \multicolumn{6}{|c|}{ Two groups (Red tilapia and others) } \\
\hline $\begin{array}{l}\text { Between } \\
\text { groups }\end{array}$ & 1 & 900.47 & -0.5567 & -1.760 & $0.639 * *$ \\
\hline $\begin{array}{c}\text { Among } \\
\text { populations } \\
\text { within groups }\end{array}$ & 5 & 4947.67 & 20.62 & 65.06 & $0.633 * *$ \\
\hline $\begin{array}{c}\text { Within } \\
\text { populations }\end{array}$ & 325 & 3781.13 & 11.63 & 36.70 & -0.018 \\
\hline Total & 331 & 9629.27 & 31.70 & & \\
\hline
\end{tabular}

3 Notes: d.f., degrees of freedom; ** means extremely significant $(P<0.01)$ 
Figure 1 (on next page)

Fig. 1 The UPGMA tree based on the D-loop sequences of seven tilapia populations

The numbers above the scale line indicate the $\mathrm{K} 2 \mathrm{P}$ genetic distances among populations . 


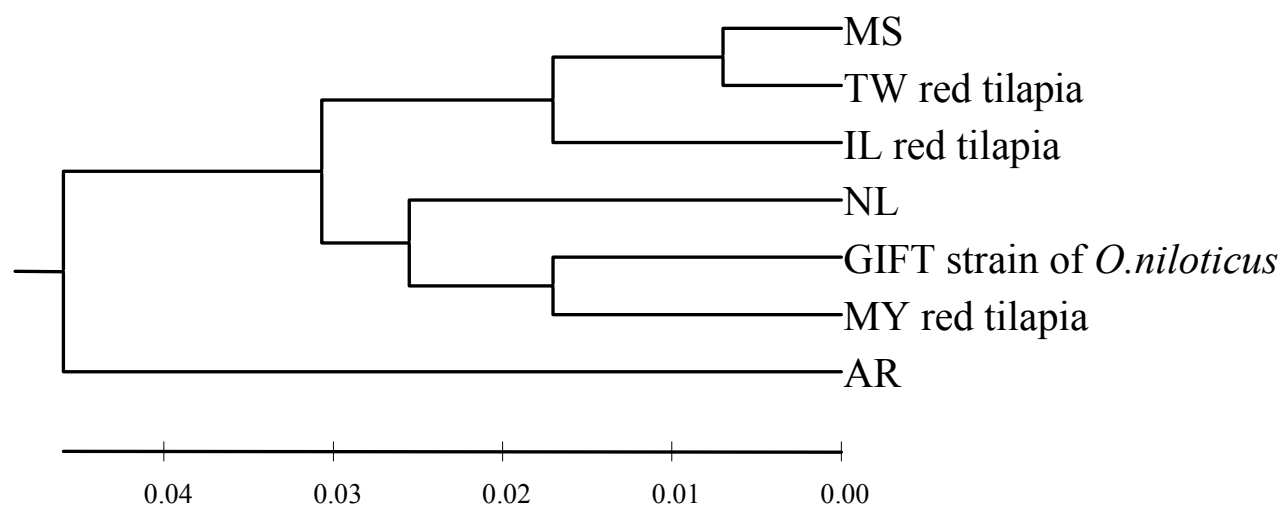


Figure 2 (on next page)

Fig.2 The haplotypes network of the D-loop sequences for seven tilapia populations.

Different populations indicated with different color; the pie sizes mean the distribution frequencies of haplotypes in populations. 
